\title{
Bio-nanorobotic in nanotechnology: structure and potential
}

\begin{abstract}
Bio-nanorobotic systems are made from bio-nanocomponents, particularly proteins. An important group of such protein-based bio-nanocomponents are myosin protein nanomotors that are involved in a wide variety of cellular tasks from cellular transports to muscle contractions. Realization of behavior and properties of the myosin protein nanomotors as bionanocomponents could form the basis of bottom-up approaches for constructing active bionanorobotic systems. This is the long-term goal of the bio-nanorobotic. In this paper we have presented an engineering point of view to the structure and behavior of both categories of myosin nanomotors, muscle and non-muscle. Myosin nanomotors are self-guiding systems. They have evolved to bind to their polymer filaments and are able to recognize the direction of movement by moving only in one direction of the polarized filaments. Myosin nanomotors also convert the chemical energy into mechanical work directly rather than via an intermediate energy. We have also pointed out to the potential applications of this nanomotor. The myosinôs perfect structure, small size, smart and high efficiency indicate that myosin nanomotor can be an ideal bio-nanocomponent for bio-nanorobotic systems.
\end{abstract}

Keyword: Bio-nanorobots; Bio-nanosystems; Nanomotors; Myosin 